\title{
Activity of upper limb and trunk muscles during power walking
}

\author{
JOHANN P. KUHTZ-BUSCHBECK | ANTONIA FRENDEL \\ Institute of Physiology, Christian-Albrechts-Universität zu Kiel \\ Correspondence to: Johann P. Kuhtz-Buschbeck, Institute of Physiology, Christian-Albrechts-Universität zu Kiel, Olshausenstraße 40, D 24098 Kiel, Germany \\ Email: kuhtz@physiologie.uni-kiel.de
}

\author{
AT A GLANCE \\ Power walking, i.e. walking with emphasized \\ arm swing, is a moderate aerobic exercise. \\ Compared to normal walking, the EMG activity \\ of upper limb muscles is tripled during power \\ walking. Similar activation patterns of some \\ muscles in both modes of walking suggest a \\ common underlying motor program. Two \\ shoulder muscles are even more active during \\ power walking than during slow running \\ (jogging).

\section{ABBREVIATIONS} \\ $A D \quad$ anterior deltoid muscle \\ BIC biceps brachii \\ EMG electromyographic \\ ES erector spinae muscle \\ LD latissimus dorsi \\ MVC maximum voluntary contraction \\ PD posterior deltoid muscle \\ TRAP trapezius muscle \\ TRI triceps brachii
}

\begin{abstract}
BACKGROUND: Power walking, i.e. walking with emphasized arm swing, increases energy expenditure. To characterize this moderate aerobic fitness exercise, the associated activation patterns of upper limb and trunk muscles need to be known. AIM: To describe the amount and pattern of EMG activity of upper limb and trunk muscles during power walking and compare it to normal walking and jogging.

METHOD: Twenty volunteers were examined on a treadmill at $6 \mathrm{~km} / \mathrm{h}$ during (a) normal walking, (b) power walking, (c) jogging. EMG data were collected for the trapezius (TRAP), anterior (AD) and posterior deltoid (PD), biceps (BIC), triceps (TRI), latissimus dorsi (LD) and erector spinae (ES) muscles.

RESULTS: Activity of four muscles (AD, BIC, PD, TRAP) was three- to fivefold stronger during power walking than normal walking $(p<0.01)$. Further significant increases $(p<0.01)$ involved the TRI, LD and ES. Two muscles $(A D, T R A P)$ were more active during power walking than jogging $(p<0.05)$. Normal walking and power walking involved similar EMG patterns of PD, LD, ES.

CONCLUSION: Emphasizing arm swing during power walking triples the EMG activity of upper limb muscles, compared to normal walking. Similar basic temporal muscle activation patterns in both modes of walking suggest a common underlying motor program.
\end{abstract}

\section{INTRODUCTION}

Power walking is a popular alternative to Nordic walking and running, which can help to maintain fitness and health. ${ }^{1}$ Beneficial effects of this moderate exercise have been demonstrated in the prevention and treatment of type 2 diabetes mellitus and in cardiac rehabilitation.2,3 Power walking has been recommended as endurance sport after total knee joint replacement, since biomechanical stress at the knee joint is lower than during jogging. ${ }^{4}$ During power walking, active upper body movements are added to the usual walking exercise (Figure 1). Arm swing is emphasized deliberately. ${ }^{5}$ With the elbows joints bent about 90 degrees, both arms swing actively back and forth (not side to side) close to the body. At the end of forward arm swing, the elbow is about level with the breastbone; at the end of backswing, the hand is near the hip. Trunk posture is upright with the back straight. The head is kept up with the chin parallel to the ground, and the eyes look straight ahead. Power walking is usually performed at gait velocities of 6 to $8 \mathrm{~km} / \mathrm{h} .1,5$

\begin{tabular}{l|l|l|l}
\hline Kuhtz-Buschbeck \& & 2015 & VOL. 9 & N.2 \\
Frendel & & &
\end{tabular}




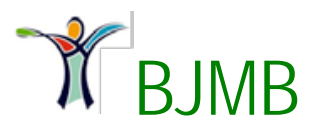

Brazilian Journal of Motor Behavior

The exercise intensities of power walking and slow running are similar. ${ }^{1}$ Knowledge about the activity of upper limb and trunk muscles during power walking is, however, limited. Two previous studies reported increased EMG activity of the biceps brachii and of leg muscles. ${ }^{6,7}$ However, to what extent are other upper limb muscles involved during power walking? Does the accentuation of arm swing engage trunk muscles, too? Do normal walking, power walking and jogging share common EMG activation waveforms, which might suggest a common underlying motor program $8,9,10$, or are they each characterized by a specific activation pattern? To answer these questions, the present study aims to provide detailed EMG data of seven upper limb and trunk muscles during walking with natural arm swing (baseline), power walking, and jogging on a treadmill. Condition-dependent changes in the amount and in the temporal pattern of EMG activity are reported. Principal component analyses (PCA) were applied to identify EMG activation patterns that remain consistent across gait conditions. ${ }^{8}$

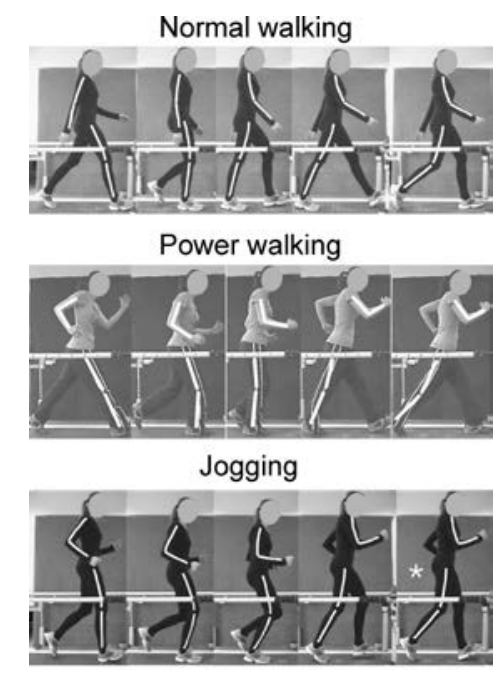

Figure 1. Gait conditions. Limb movements in the three gait conditions, each illustrated with video frames of a stance phase of the right leg (ipsilateral to EMG electrodes). Normal walking: baseline condition with natural arm swing. Power walking: arm swing is accentuated deliberately. Jogging: The stance phase is followed by a short flight phase (asterisk) without foot contact to the ground. Treadmill velocity is always $6 \mathrm{~km} / \mathrm{h}$.

\section{METHODS}

\section{Participants}

Twenty healthy volunteers ( 10 women) participated. Their mean age was 26 years (SD 6 years), their average body height $177 \pm 8 \mathrm{~cm}$ and their weight $72 \pm 11 \mathrm{~kg}$ (mean \pm SD). Exclusion criteria were any orthopedic or neurological pathology affecting gait and/or balance or any apparent gait abnormality. Most of the volunteers were sportive college students. All were familiar with walking and jogging on a treadmill, but not accustomed to power walking prior to the experiments. They wore tank tops, sweatpants and their personal running shoes. Written consent for participation and publication was obtained. 
The local Ethical Committee (Christian Albrechts University, Kiel, Germany) approved the procedures.

\section{Task and Instrumentation}

We used a treadmill (Woodway ${ }^{\circledR}$, Germany) with a horizontal belt surface $(162 \mathrm{~cm}$ long, $44 \mathrm{~cm}$ wide) and with the handrails set $1 \mathrm{~m}$ apart. A thin string connected the volunteers to a magnet-operated emergency stop switch. In randomized order, each subject performed three gait conditions, namely (i) normal walking with free arm swing (baseline), (ii) slow running, i.e. jogging, and (iii) power walking (Figure 1). In an upright body posture, both arms moved briskly back and forth close to the body during power walking, with the elbows maintaining an angle of around 90 degrees. ${ }^{5}$ Initially, detailed instructions, video clips, and about 15 minute of practice made the participants familiar with power walking. Performance was smooth when EMG recordings started. The gait conditions were examined in trials of $\sim 80 \mathrm{~s}$ duration, with EMG data recorded continuously throughout the last minute of each trial. Stance and swing phases of the right leg (foot contacts) were recorded with optical sensors mounted on the treadmill (Optogait $\circledast$ virtual footswitch, Bolzano, Italy). All gait trials were videotaped and checked for correct performance. In case of irregularities (e.g. tripping, hand gestures, grasping the handrail), the respective gait trial was repeated. Since the amount of EMG activity changes with gait speed ${ }^{11,12}$, the same treadmill velocity was used $(6 \mathrm{~km} / \mathrm{h})$ in all three gait conditions, as a prerequisite for later comparisons of the respective EMG amplitudes. The chosen velocity is appropriate for walking, power-walking and jogging. 1,5

Disposable EMG electrodes (Arbo® H124SG, Germany; $\mathrm{Ag}-\mathrm{AgCl}$, pick-up diameter $15 \mathrm{~mm}$ ) were attached to the abraded skin above seven muscles on the right side of the body in a bipolar configuration, with an inter-electrode distance of $\sim 2.5 \mathrm{~cm}$. In line with published guidelines ${ }^{13}$, pairs of electrodes were placed on the upper trapezius muscle (TRAP), anterior deltoid (AD), posterior deltoid (PD), biceps brachii (BIC), long head of the triceps brachii (TRI), on the (LD) caudal of the inferior scapular angle, and on the erector spinae (ES), electrodes were placed $\sim 3 \mathrm{~cm}$ lateral of vertebra $L 3$ spinous process. More detailed information concerning electrode placement has been published elsewhere. ${ }^{14}$

EMG signal amplitudes were normalized to the highest signal reached during a maximal voluntary contraction (\% MVC) to account for variations of the absolute EMG voltages between different muscles and individuals ${ }^{15}$, which may be due to differences in skin conductance. Maximum voluntary contractions were therefore performed with each relevant muscle prior to the gait trials. The TRAP was tested by abducting the extended arm in the frontal plane. Anterior and posterior deltoid muscles (AD, PD) and LD were tested by vigorous flexion/extension of the shoulder against fixed resistance, with the elbow extended. TRI and BIC contracted maximally against resistance, with the elbow flexed by $\sim 90^{\circ}$. To test the paravertebral erector spinae (ES), the participants lay prone and then extended the trunk forcefully against resistance.

The EMG electrodes were connected via pre-amplified electrode leads to an eightchannel Myosystem 1400 L (Noraxon®, Scottsdale, AZ, USA) EMG system with the following specifications: differential amplifier, input impedance $>100 \mathrm{MOhm}$, common mode rejection ratio $>100 \mathrm{~dB}$ at $60 \mathrm{~Hz}$, sensitivity $1 \mu \mathrm{V}$, baseline noise $<1 \mu \mathrm{V} R \mathrm{RMS}$, bandwidth $10-500 \mathrm{~Hz}$. EMG signals of the seven muscles were sampled at a rate of 1000 
$\mathrm{Hz}$ per channel and A/D converted with a digital 12 bit resolution per channel. One channel was used for footswitch data. All data were stored together with video images for later offline processing using Noraxon® software (Myo Research XP, Master Edition 1.07).

\section{Data Analysis}

EMG data of each muscle were filtered digitally (FIR bandpass 10-250 Hz), fullwave rectified, smoothed with a root mean square window of $50 \mathrm{~ms}$, and amplitudenormalized (\% MVC) to the highest activity level (mean amplitude for 0.3 seconds) measured in the set of maximum voluntary contractions. ${ }^{15}$ As a common time frame, the gait cycle of the right leg (ipsilateral to the muscles investigated) was time-normalized from $1 \%$ (onset of stance, heelstrike of right foot) to $100 \%$ (end of swing phase of right leg). EMG data of $\sim 50$ gait cycles per subject and condition were averaged to obtain typical individual EMG profiles. From these, ensemble averaged EMG curves (group results) were obtained. To describe the mean activation of each muscle, the EMG amplitude was averaged across the entire gait cycle in each subject and condition. To detect (withinsubject) differences in muscle activation between the three conditions (power walking, jogging, normal walking), analyses of variance were performed (ANOVA), followed by paired t-tests at the 0.05 significance level. In each subject, we also calculated the relative (percentage) increase of the EMG signal amplitudes during power walking and jogging above the baseline EMG activity of normal walking (=100 \%).

From EMG curves of a larger number of muscles, principal components analyses (PCA) extract a smaller number of underlying basic temporal activation patterns (factor scores), which account for most of the total variance of the original data set. ${ }^{16,17}$ PCA was applied to the ensemble averaged EMG curves, i.e. to one matrix $(m \times t)$ per condition, with $m=7$ (number of muscles) and $t=100$ (normalized 100-point time base of gait cycle). We used the statistics software SPSS IBM version 17 (New York, USA); module data reduction/factor analysis/extraction of principal components. The steps include calculation of the correlation matrix, extraction of the initial principal components, computation of eigenvalues, varimax rotation of the factors, calculation of the factor scores (basic temporal components) and factor loadings (weighting coefficients). The factor loadings indicate how a given factor score is distributed to different muscles. ${ }^{17}$ Factor scores with eigenvalues greater than unity were retained. ${ }^{18}$ Correlations between the factor scores of the three gait conditions (normal walking, power walking, jogging) were analyzed by calculating Pearson's correlation coefficients ( $r$ ).

\section{RESULTS}

Averaged EMG curves of normal walking (baseline), power walking and jogging are displayed in Figure 2, and the corresponding mean EMG amplitudes are listed in Table 1. All investigated muscles were significantly $(p<0.01)$ more active during power walking than normal walking. EMG amplitudes of TRAP, PD, BIC, AD changed the most, with percentage increases of $+200 \%$ to $+450 \%$ above the baseline of normal walking $(=100 \%)$. Across all seven investigated muscles, EMG activity about tripled during power walking 
(mean change $+208 \%$ ). Also during jogging, the EMG activity of all muscles increased significantly $(p<0.01)$ above baseline level (Table 1$)$, with a mean percentage change of $+130 \%$ across muscles. Two muscles (TRAP, AD) were significantly more active during power walking than during jogging $(p<0.05)$, while reverse differences did not reach significance.

Condition-dependent differences of the EMG patterns are evident from Figure 2. During power walking, the TRAP was nearly continuously active throughout the gait cycle. During jogging, however, this muscle showed notable biphasic activity, with the two maxima occurring around $10 \%$ and $60 \%$ of the gait cycle, shortly after heelstrike of each foot. A marked peak of $A D$ activity occurred at $\sim 90 \%$ of the gait cycle during power walking only. At that time, backward swing (shoulder extension) of the arm reverses to forward arm swing (shoulder flexion). Such peak $A D$ activity was not found during normal walking or jogging. In all three gait conditions, peak PD activity was reached around the middle of the gait cycle. This maximum coincides with heel strike of the contralateral left foot (see rightmost video frames in Figure 1), and with the reversal from forward arm swing to backward arm swing. An earlier (around time 15\%) and smaller peak of the PD signal occurred during jogging, but not in either walking condition. BIC and TRI co-contracted during power walking, with some modulations of their EMG amplitudes across the gait cycle. On the other hand, BIC was hardly active during normal walking, and showed a marked biphasic EMG pattern during jogging, which matched the TRAP activation waveform. The EMG curves of LD and ES during normal walking and power walking were very similar, with marked peaks in the middle of the gait cycle (time $\sim 50 \%$ ). Conversely, EMG waveforms of these muscles differed during jogging (Figure 2). Here LD activity started in the early stance phase (around time 10\%) and continued until the swing phase of the ipsilateral leg (time $\sim 60 \%$ ). Forward arm swing and shoulder flexion during the stance phase of jogging (see Figure 1) were hence associated with lengthening (eccentric) LD contractions. The ES was mainly active throughout the second half of the gait cycle during jogging.

From the EMG curves of the seven muscles, PCA extracted two factors with eigenvalues greater than unity $(A, B)$ for each gait condition. Figure 3 depicts the respective factor scores $A$ and $B$, i.e. the basic temporal activation components. They accounted for between $81 \%$ and $93 \%$ of the total EMG waveform variance. Factor scores A of normal walking, power walking and jogging had different shapes, as indicated by correlation coefficients $r$ ranging between -0.55 and 0.4 . The factor loadings indicate how a given component is allocated to the different muscles. Muscles that loaded highly on component $A$ were TRAP, AD, BIC for normal walking; AD, BIC and TRI for power walking, and TRAP, AD, BIC for jogging. The factor scores $B$ of normal walking and power walking were nearly congruent (correlation coefficient $r=0.98$ ), as were the muscles loading on this factor (PD, LD, ES). Hence, despite conspicuous condition-dependent differences in the amount of EMG activity (Table 1), these three muscles exhibited very similar temporal activation profiles during normal walking and power walking. Conversely the activation component $B$, derived from EMG data of jogging, differed from the components $B$ that typified walking (correlation coefficients $r<0.65$ ). Muscles that loaded on component $B$ during jogging were PD, TRI and LD. 
Compared to normal walking at the same speed $(6 \mathrm{~km} / \mathrm{h})$, the volunteers took longer steps and had a lower cadence (step frequency) during power walking. The average step lengths were $82.8 \pm 4.4 \mathrm{~cm}$ for normal gait (mean \pm SD) and $85.1 \pm 5.6 \mathrm{~cm}$ for power walking. The corresponding values of the cadence were $121.1 \pm 6.4$ steps per minute (normal walking) and $117.9 \pm 7.5$ steps / min (power walking). These differences between walking conditions were significant (t-tests, $p<0.05$ ).

Table 1: EMG activity levels during normal walking, jogging, and power walking

\begin{tabular}{|c|c|c|c|c|c|c|}
\hline $\begin{array}{l}\text { Muscles } \\
\text { (right side) }\end{array}$ & & $\begin{array}{c}\text { Normal walking } \\
(6 \mathrm{~km} / \mathrm{h})\end{array}$ & $\begin{array}{l}\text { Jogging } \\
(6 \mathrm{~km} / \mathrm{h})\end{array}$ & & $\begin{array}{c}\text { Power walking } \\
(6 \mathrm{~km} / \mathrm{h})\end{array}$ & $\begin{array}{c}\text { ANOVA } \\
\mathrm{F} 2,38 \text { values }\end{array}$ \\
\hline TRAP & $\begin{array}{l}\text { EMG amp } \\
\% \text { change }\end{array}$ & $\begin{array}{c}3.55 \pm 1.86= \\
100 \%\end{array}$ & $\begin{array}{c}6.88 \pm 2.45 \text { ** } \\
+119 \pm 77 \%\end{array}$ & \# & $\begin{array}{l}9.08 \pm 4.66 \text { ** } \\
+205 \pm 230 \%\end{array}$ & 23.81 \\
\hline$A D$ & $\begin{array}{l}\text { EMG amp. } \\
\% \text { change }\end{array}$ & $\begin{array}{c}0.8 \pm 0.43= \\
100 \%\end{array}$ & $\begin{array}{c}1.37 \pm 0.73^{* *} \\
+78 \pm 52 \%\end{array}$ & $\#$ & $\begin{array}{l}3.87 \pm 3.32^{* *} \\
+449 \pm 461 \%\end{array}$ & 14.85 \\
\hline PD & $\begin{array}{l}\text { EMG amp. } \\
\% \text { change }\end{array}$ & $\begin{array}{c}2.49 \pm 0.97= \\
100 \%\end{array}$ & $\begin{array}{c}6.20 \pm 3.23^{\star *} \\
+146 \pm 72 \%\end{array}$ & & $\begin{array}{l}8.05 \pm 6.12 \text { * } \\
+232 \pm 221 \%\end{array}$ & 12.45 \\
\hline $\mathrm{BIC}$ & $\begin{array}{l}\text { EMG amp. } \\
\% \text { change }\end{array}$ & $\begin{array}{l}0.63 \pm 0.21 \\
=100 \%\end{array}$ & $\begin{array}{l}2.33 \pm 1.22 \text { * } \\
+263 \pm 240 \%\end{array}$ & & $\begin{array}{l}2.57 \pm 1.54 \text { ** } \\
+298 \pm 240 \%\end{array}$ & 28.29 \\
\hline TRI & $\begin{array}{l}\text { EMG amp. } \\
\% \text { change }\end{array}$ & $\begin{array}{c}1.50 \pm 0.92 \\
=100 \%\end{array}$ & $\begin{array}{c}2.90 \pm 1.93^{* *} \\
+91 \pm 42 \%\end{array}$ & & $\begin{array}{l}3.18 \pm 1.83^{* *} \\
+126 \pm 125 \%\end{array}$ & 15.09 \\
\hline LD & $\begin{array}{l}\text { EMG amp. } \\
\% \text { change }\end{array}$ & $\begin{array}{c}3.30 \pm 1.42= \\
100 \%\end{array}$ & $\begin{array}{c}7.45 \pm 3.32 \text { * } \\
+139 \pm 67 \%\end{array}$ & & $\begin{array}{c}5.78 \pm 3.01 \text { * } \\
+88 \pm 92 \%\end{array}$ & 18.10 \\
\hline ES & $\begin{array}{l}\text { EMG amp. } \\
\% \text { change }\end{array}$ & $\begin{array}{c}7.2 \pm 2.52= \\
100 \%\end{array}$ & $\begin{array}{c}12.55 \pm 5.76 \text { ** } \\
+76 \pm 57 \%\end{array}$ & & $\begin{array}{c}11.19 \pm 4.06 \text { ** } \\
+58 \pm 39 \%\end{array}$ & 19.81 \\
\hline
\end{tabular}

EMG amp.: Mean EMG amplitude, averaged over the entire gait cycle. All EMG amplitudes are normalized to maximum voluntary contractions [unit \% MVC]. Numbers represent inter-individual mean \pm standard deviation. $\%$ change: EMG activity of normal walking has been set to $100 \%$ (baseline) to calculate percentage increases for the other conditions. Muscles: trapezius TRAP, anterior deltoid AD, posterior deltoid PD, biceps brachii BIC, triceps brachii TRI, latissimus dorsi LD, erector spinae ES.

** EMG amplitude is significantly ( $p<0.01$; paired t-tests) higher than during normal walking (baseline)

\# Significant difference between power walking and jogging with $p<0.05$ (paired t-test) 


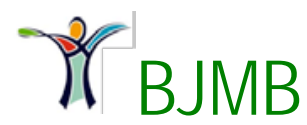

Brazilian Journal of Motor Behavior

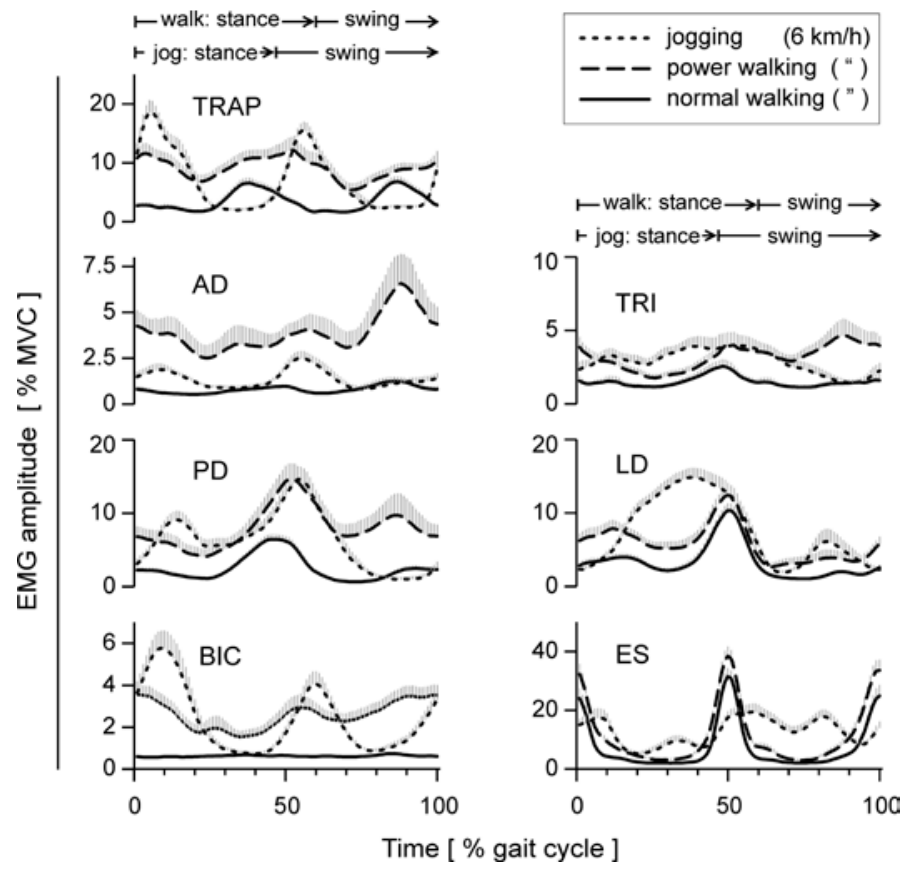

Figure 2. EMG curves of the three gait conditions. Muscle activation curves during normal walking (solid line), power walking (broken line) and jogging (dotted line). Ensemble averaged EMG curves (inter-individual mean of the 20 subjects) of each condition, with thin vertical lines indicating inter-individual variability (standard error of the mean). Treadmill velocity was always $6 \mathrm{~km} / \mathrm{h}$. Stance and swing phases of the right leg are indicated above. For abbreviations of muscles see Table 1.

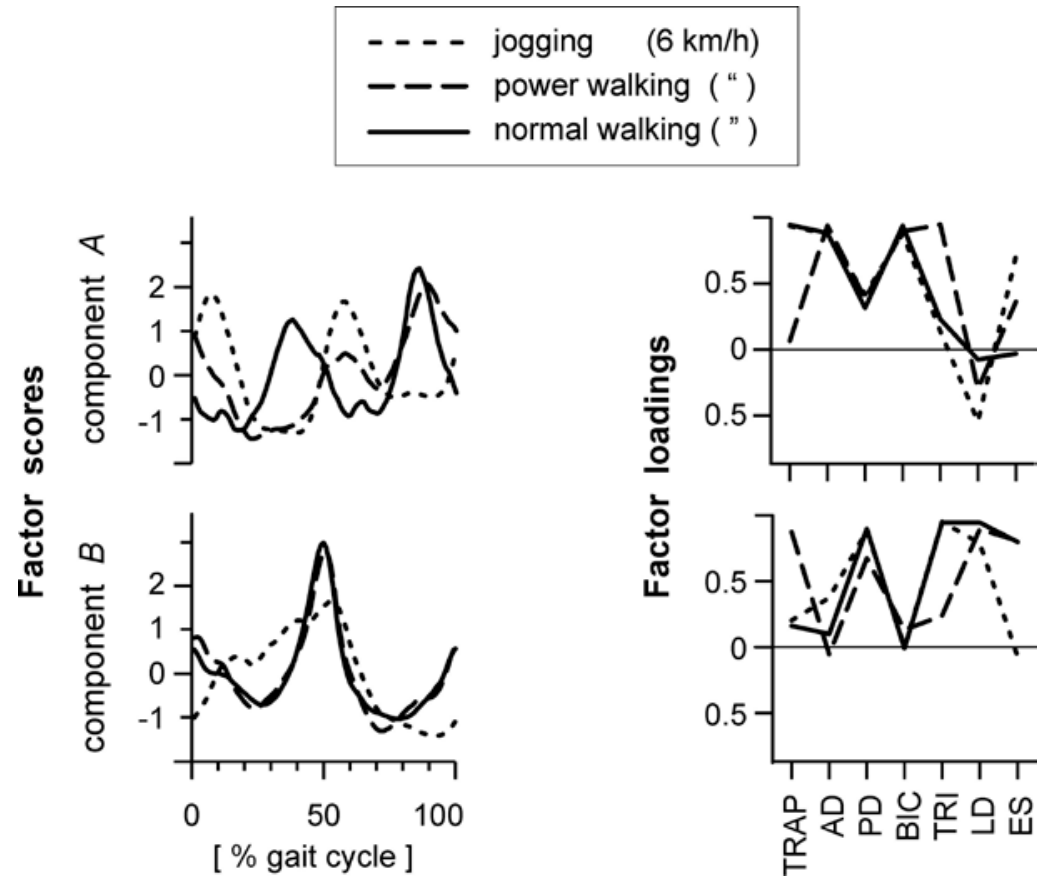

Figure 3. Basic temporal muscle activation components. From the EMG curves of each gait condition, two temporal activation components $A$ and $B$ (factor scores) with eigenvalues greater than unity were derived by principal component analysis (PCA). The factor scores of the three conditions are superim-posed for comparison. Factor loadings indicate how a given factor score is allocated to the different muscles. Data of normal walking (solid line), power walking (broken line), jogging (dotted line). For abbreviations of muscles see Table 1. 


\section{DISCUSSION}

Natural arm swing is not a purely passive movement, but involves shortening and lengthening contractions of arm and shoulder muscles. ${ }^{11,14,19}$ Arm swing improves gait stability and decreases angular momentum of the body about the vertical axis. ${ }^{20,21,22}$ The deliberate accentuation of arm swing during power walking is known to augment energy expenditure. Park and colleagues ${ }^{1}$ studied twenty healthy young male volunteers during power walking and running at the same velocity $(6.7 \mathrm{~km} / \mathrm{h})$ on a treadmill. Oxygen consumption ( $27 \mathrm{ml} / \mathrm{kg}$ body weight per minute) and heart rate $(\sim 145$ beats $/ \mathrm{min})$ during power walking were comparable to the values measured during running (oxygen uptake $\sim 29 \mathrm{ml} / \mathrm{kg}$ per minute, heart rate $\sim 150$ beats/min). The authors concluded that the exercise intensities of power walking and jogging are similar and suggested power walking as a good alternative aerobic exercise.

It is conceivable that upper arm muscles acting on the shoulder and elbow joints are involved since arm swing is emphasized during power walking. In congruence, Cho and colleagues reported enhanced EMG activity of the M. biceps brachii. ${ }^{6}$ Our results confirm and extend their findings with EMG patterns of other upper limb muscles. The strongest relative changes, compared to normal gait, involved the anterior deltoid (AD, fivefold increase) and biceps (BIC, fourfold increase), followed by the posterior deltoid and trapezius (PD, TRAP, threefold increases), triceps and latissimus dorsi (TRI, LD, about twofold increases). Hence not only flexors (AD, BIC), but also extensors of the shoulder (LD, TRI) and elbow joints (TRI) were engaged during power walking. Two muscles (AD, TRAP) were even more active during power walking than jogging, while reverse differences were not found. Note that the baseline level influences the relative changes, i.e. they will be amplified when the baseline activity is low. Still, the absolute increases of the EMG amplitudes above baseline levels during power walking were all significant $(p<0.01$; see Table 1).

Interestingly, also trunk muscles were involved, as the ES was significantly $(p<0.01)$ more active during power walking than in normal gait. ES activity may stabilize the pelvis when body weight is transferred from one leg to the other leg. ${ }^{23}$ Functional neural coupling between arms and trunk could play a role, too. Recent research demonstrated neural coupling between arms and legs with experiments where rhythmical arm movements enhanced and shaped muscle activity of the legs. ${ }^{24}$ Similarly, two EMG studies reported enhanced activity of the quadriceps, biceps femoris, tibialis anterior and gastrocnemius/soleus muscles during power walking, possibly with a more powerful plantar flexion of the feet at the end of stance.6,7 Correspondingly, our data show a significant increase of the step length during power walking, while the cadence was decreased, and an involvement of trunk muscles.

Three muscles (PD, LD, ES) exhibited virtually the same temporal activation pattern during power walking and normal walking. PD and LD activations reached their peaks in the middle of the gait cycle, around heelstrike of the contralateral foot (Fig. 3, component B). Since both muscles retract the upper arm, their contractions will decelerate forward arm swing and initiate backward swing. ${ }^{11,19}$ Both walking conditions share common 
biomechanical features, such as an inverted pendulum mechanism, where the center of mass of the body vaults over the supporting leg during the stance phase, and short double support phases, where both feet are in contact with the ground. ${ }^{25}$ Distribution of one basic temporal activation pattern to multiple muscles in different modes of gait represents an efficient neuronal control strategy. 8,17

Four other muscles (TRAP, BIC, TRI, AD) had specific temporal activation patterns during power walking, which differed from normal walking. The nearly continuous activation of the TRAP during power walking may control shoulder inclination in the frontal plane and shoulder abduction, so that the arms swing vigorously in anterior-posterior direction and not in front of the body. ${ }^{12}$ Co-activation of BIC and TRI may help to stabilize the elbow joint in a flexed posture $\left(\sim 90^{\circ}\right)$. At the end of the swing phase of the ipsilateral leg, strong $A D$ activity guides the reversal from backward to forward arm swing, i.e. from shoulder extension to shoulder flexion during power walking. Local minima of the TRAP, AD, BIC and TRI activation curves around midstance of the ipsilateral leg ( $30 \%$ of the gait cycle) suggest that forward arm swing is facilitated by passive dynamics at that time.

There are limitations to our study, which might be considered in related future research. Since the participants were initially not familiar with power walking, they rehearsed until their performance was smooth, and then EMG data recordings started. Familiarity with the task might still be a confounding factor. Due to technical constraints, kinematic and leg muscle EMG data could not be obtained simultaneously with upper limb muscle EMG signals. The number of available EMG channels was restricted to seven. $A$ treadmill was used for practical reasons (constant gait velocity, no EMG telemetry available), although over ground and treadmill locomotion may differ to some extent.

\section{CONCLUSION}

Walking with emphasized arm swing (power walking) is a moderate aerobic exercise. Compared to normal gait, the EMG activity of upper limb muscles is tripled. Also trunk muscles are more active during power walking. Similar EMG patterns in both modes of walking suggest a common underlying motor program.

\section{ACKNOWLEDGEMENTS}

We thank the volunteers for their patience and willingness to take part in the experiments. The help of Martin Müller in preparation of the treadmill is gratefully acknowledged.

\section{REFERENCES}

1. Park DS, Nam HS, Kim DH, Her JG, Woo JH. The comparison of exercise intensity between power walking and running. Korean Journal of Sports Medicine 2008; 26(2): 175-79. [in Korean] 


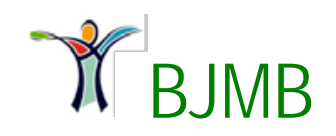

Brazilian Journal of Motor Behavior

2. Kurban S, Mehmetoglu I, Yerlikaya HF, Gönen S, Erdem S. Effect of chronic regular exercise on serum ischemia-modified albumin levels and oxidative stress in type 2 diabetes mellitus. Endocr Res 2011; 36(3): 116-23.

3. Kim C, Kim BO, Lim KB, Kim YJ, Park YB. The effect of power-walking in phase 2 cardiaC rehabilitation program. Ann Rehabil Med 2012; 36(1): 133-40.

4. Kuster MS, Spalinger E, Blanksby BA, Gächter A. Endurance sports after total knee replacement: a biomechanical incestigation. Med Sci Sports Exerc 2000; 32(4): 721-24.

5. Meakin J. The beginner's guide to power walking. Hauppauge, NY, 2003: Barron's Educational Series.

6. Cho KK, Kim YS, Kim EJ. The comparative analysis of kinematic and EMG on power walking and normal gait. Korean Journal of Sport Biomechanics 2006; 16: 85-95. [in Korean] 7. Gi SJ, Chae WS, Kang NJ, Jang JI, Yoon CJ. The comparative analysis of EMG activities on the lower limb muscles during power walking and normal walking. Korean Journal of Sport Biomechanics 2008; 18(4): 125-33. [in Korean]

8. Lacquaniti F, Ivanenko YP, Zago M. Patterned control of human locomotion. J Physiol 2012; 590: 2189-99.

9. Zelik KE, La Scaleia V, Ivanenko YP, Lacquaniti F. Can modular strategies simplify neural control of multidirectional human locomotion? J Neurophysiol 2014; 111: 1686-1702.

10. Ivanenko YP, Poppele RE, Lacquaniti F. Distributed neural networks for controlling human locomotion. Lessons from normal and SCI subjects. Brain Res Bull 2009; 78: 13-21.

11. Kuhtz-Buschbeck JP, Jing B. Activity of upper limb muscles during human walking. J Electromyogr Kinesiol 2012; 22: 199-206.

12. Hogue RE. Upper-extremity muscular activity at different cadences and inclines during normal gait. Phys Ther 1969; 49: 963-72.

13. Hermens HJ, Freriks B, Disselhorst-Klug C, Rau G. Development of recommendations for SEMG sensors and sensor placement procedures. J Electromyogr Kinesiol 2000; 10: 361-74.

14. Kuhtz-Buschbeck JP, Frendel A. Stable patterns of upper limb muscle activation in different conditions of human walking. BJMB 2015; 9(1): 1-10.

15. Burden A. How should we normalize electromyograms obtained from healthy participants? What we have learned from over 25 years of research. J Electromyogr Kinesiol 2010; 20: 1023-35.

16. Chau T. A review of analytical techniques for gait data. Part 1: Fuzzy, statistical and fractal methods. Gait Posture 2001; 13: 49-66.

17. Ivanenko YP, Poppele RE, Lacquaniti F. Five basic muscle activation patterns account for muscle activity during human locomotion. J Physiol 2004; 556: 267-82.

18. Davis BL, Vaughan CL. Phasic behavior of EMG signals during gait: use of multivariate statistics. J Electromyogr Kinesiol 1993; 3: 51-60.

19. Goudriaan M, Jonkers I, van Dieen JH, Bruijn SM. Arm swing in human walking: What is their drive? Gait Posture 2014; 40: 321-26. 


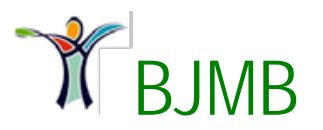

Brazilian Journal of Motor Behavior

20. Collins SH, Adamczyk PG, Kuo AD. Dynamic arm swinging in human walking. Proc R Soc Lond B Biol Sci 2009; 276: 3679-88.

21. Pontzer H, Holloway JH, Raichlen DA, Lieberman DE. Control and function of arm swing in human walking and running. J Exp Biol 2009; 212: 523-34.

22. Meyns P, Bruijn SM, Duysens J. The how and why of arm swing during human walking. Gait Posture 2013; 38: 555-62.

23. Ceccato JC, de Sèze M, Azevedo C, Cazalets JR. Comparison of trunk activity during gait initiation and walking in humans. PLoS ONE 2009; 4: e8193.

24. Sylos-Labini F, Ivanenko YP, MacLellan MJ, Cappellini G, Poppele RE, Lacquaniti F. Locomotor-like leg movements evoked by rhythmic arm movements in humans. PLOS ONE 2014; 9(3): e90775.

25. Cappellini G, Ivanenko YP, Poppele RE, Lacquaniti F. Motor patterns in human walking and running. J Neurophysiol 2012; 95: 3426-37.

Citation: Kuhtz-Bushbeck \& Frendel. Activity of upper limb and trunk muscles during power walking. BJMB. 2015: 9(2): 1-12.

Editor: Joao A. C. Barros, California State University Fullerton, Fullerton, CA, USA.

Copyright: $\bigcirc 2015$ Kuhtz-Bushbeck \& Frendel and BJMB. This is an open-access article distributed under the terms of the Creative Commons Attribution-NonCommercial-NoDerivatives 4.0 International License which permits unrestricted use, distribution, and reproduction in any medium, provided the original author and source are credited.

Competing interests: The authors have declared that no competing interests exist.

Funding: There was no funding for this study

Download: http://socibracom.com/bjmb/index.php/bjmb/issue/view/25 